\title{
BP-OP-2-6
}

\section{The triple-checked criteria for drain management after pancreatectomy}

\author{
Daisuke HASHIMOTO, Sohei SATOI*, Tomohisa YAMAMOTO, So YAMAKI, Satoshi HIROOKA, Tatsuma SAKAGUCHI, Mitsugu SEKIMOTO
}

Department of Surgery, Kansai Medical University, Osaka, Japan

Introduction: Management of drains is important for the detection and treatment of clinically relevant postoperative pancreatic fistula (CR-POPF) after pancreatectomy. In 2016, we established the triple-checked criteria to remove drains after pancreatectomy: drain fluid amylase (DFA) $<5,000 \mathrm{U} / \mathrm{L}$ on POD 1 and DFA $<3,000 \mathrm{U} / \mathrm{L}$ on POD 3, or CRP $<15 \mathrm{mg} / \mathrm{dL}$ on POD 3 . The aim of this retrospective study was to validated the efficacy and safety of the triple-checked criteria.

Methods: Six hundred eighty-one patients who underwent pancreatectomy from 2012 to 2019 were included. Drains were removed following our previous criteria (sequentially-checked criteria: DFA $<5,000 \mathrm{U} / \mathrm{L}$ on POD 1 and DFA $<3,000$ U/L on POD 3) from 2012 to 2016 (control group), and following the triple-checked criteria from 2017 to 2019 (intervention group).

Results: The control group included 406 patients (256 PD, 142 DP and 8 others), and the intervention group included 275 patients (177 PD, 93 DP and 5 others) ( $p=0.938)$. Pancreatic texture was soft in $68.2 \%$ patients in the control group and $62.5 \%$ patients in the intervention group $(p=0.125)$. In the control group, $76.1 \%$ patients met the sequentially-checked criteria. On the other hand, significantly more patients $(83.6 \%)$ met the triple-checked criteria in the intervention group $(p=0.018)$. There was no significant difference in the incidence of CR-POPF (11.1\% vs. $14.2 \%, p=0.228)$. False positive rate of the criteria was significantly lower in the intervention group (31.1\%) than in the control group $(64.9 \%, p<0.001)$.

Conclusions: Triple-checked criteria contributed to safe drain removal after pancreatectomy without increasing CR-POPF. 